\title{
Ground water quality assessment of different educational institutions in Rajshahi city corporation, Bangladesh
}

\author{
Abdullah Al Masum*, Md. Zahanggir Alam, Raihan Khan Opu, Anupam Chowdhury \\ Dept. of Civil Engineering, Rajshahi University of Engineering \& Technology, Rajshahi, Bangladesh \\ Email address: \\ masum24706@gmail.com (A. A. Masum), zahanggir_00ce@yahoo.com (M. Z. Alam), raihan.ce08@gmail.com (R. K. Opu), \\ anupam_19ce@yahoo.com (A. Chowdhury)
}

\section{To cite this article:}

Abdullah Al Masum, Md. Zahanggir Alam, Raihan Khan Opu, Anupam Chowdhury. Ground Water Quality Assessment of Different Educational Institutions in Rajshahi City Corporation, Bangladesh. American Journal of Environmental Protection. Vol. 3, No. 2, 2014, pp. 64-67. doi: 10.11648/j.ajep.20140302.14

\begin{abstract}
A study to assess the quality of drinking water is very essential for knowing whether the various impurities of water should be within allowable limit. Ground water samples from different educational institutions situated in Rajshahi City Corporation have been analyzed in order to check its quality especially for drinking purposes. The various important qualities of water such as $\mathrm{pH}$, iron, manganese, chlorides, arsenic, total solids, dissolved solids, suspended solids, total acidity, total alkalinity, $\mathrm{BOD}, \mathrm{COD}, \mathrm{DO}$, color, turbidity, hardness, $\mathrm{CO}_{2}$ of water, fecal and total coliform were tested. The values of all parameters except $\mathrm{pH}$ and total acidity in water samples collected from all locations were found satisfactory. In supply water of these educational institutions contain high concentration of DO, COD, total and fecal coliform, electric conductivity. These qualities of water cause harmful effect on human health. Water qualities of Rajshahi Polytechnique Institute, Rajshahi College and Rajshahi Govt. Degree College were observed satisfactory compared to other institutions.
\end{abstract}

Keywords: Educational Institutions, Ground Water, Rajshahi City Corporation, Surface Water, Water Quality, BOD, COD

\section{Introduction}

Water is the most remarkable commodity. It is absolute essential to all lives both animal and plants. It is difficult to imagine any environment without water. Man can live only three or four days without water. Potable and drinking water plays an important role in the development of good health condition of a nation. Water pollution is one of the major problems in Bangladesh. Polluted water is responsible for water borne diseases. Water obtained from different sources is associated with a large number of impurities [1]. The presence of heavy metals also plays toxic roles to human health. Many of them exert cumulative effects. The impurities present in natural water are namely, living impurities, organic impurities and water borne microorganism. The mineral impurities are mainly dissolved gasses and carbonates, sulfates, sulfide etc. On sodium, magnesium, iron etc. Inadequate quantities of water for the maintenance of personal hygiene and environmental sanitation have been shown to be the major contributions factors in the spread of epidemic diseases. The World Health Organization (WHO) states that water containing many types of impurities from various sources causes different types of water borne diseases and each year millions of people suffer from such diseases due to unsafe drinking water supplies. The aim of the present study was to assess the quality of drinking water uplifted from hand tube wells, shallow tube wells and deep tube wells in different educational institution in Rajshahi City Corporation area.

\subsection{Study Area}

Rajshahi, the fourth largest city of Bangladesh, lies between $24^{\circ} 21^{\prime}$ and $24^{\circ} 25^{\prime}$ North latitudes and between $88^{\circ} 32^{\prime}$ and $88^{\circ} 40^{\prime}$ East longitudes, with an area of about $96.68 \mathrm{sq}$. km. It is surrounded by two major outfall rivers, namely the Padma and the Barnai. The Padma is situated at the southern border and the Barnai is about $6 \mathrm{~km}$ away from the northern edge of the city.

\section{Methodology}

\subsection{Water Sample Collection}

To investigate the water quality of different educational institutions samples were collected from selected locations where location A=Rajshahi University (RU), B=Rajshahi University of Engineering \& Technology (RUET), 
$\mathrm{C}=$ Rajshahi Medical College (RMC), D=Rajshahi Govt. Degree College (RGDC), E=Rajshahi Polytechnique Institute (RPI) and F=Rajshahi college (RC). The collected samples were tested in Public Health Engineering Lab, RUET by standard method and analyzed them subsequently.

\subsection{Water Sample Technique}

22 ground water samples of 2 litres each from the selected locations throughout the area were collected in dry clean plastic containers. Before collecting the ground water samples, the wells were pumped for $4 / 5$ minutes to obtain the fresh ground water and then after rinsing the containers with pumped fresh water, the containers were filled up. Aeration was avoided as far as possible during sampling. Samples collected from the study area were carefully transported to the laboratory and preserved at a cool place in the dark.

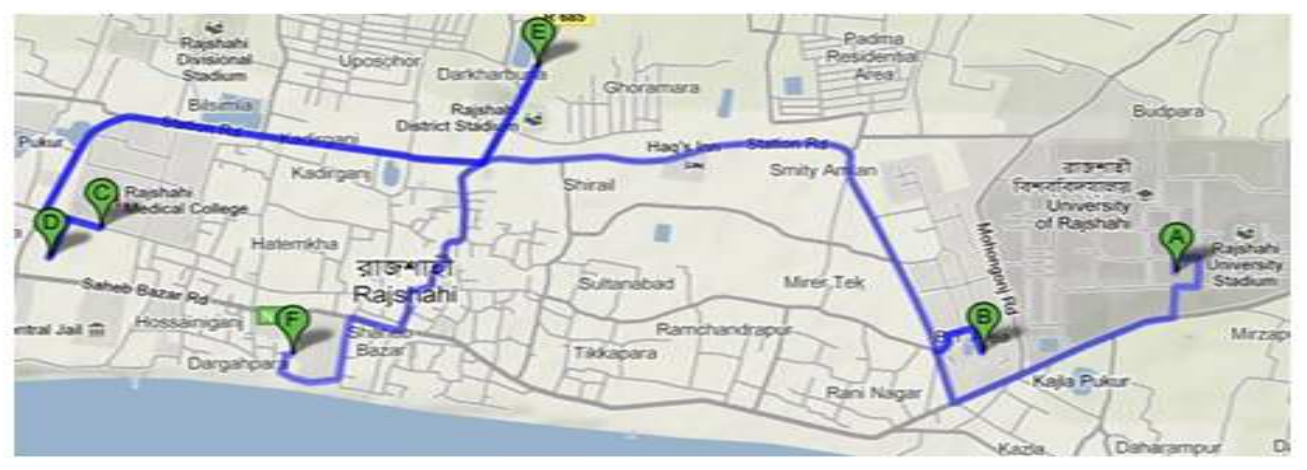

Figure 1. Location map of the study area.

Table 1. Sample Location.

\begin{tabular}{lll}
\hline Sl. No. & Sample Name & Location \\
\hline 1 & S1 & Shahid Abdul Hamid Hall, RUET \\
2 & S2 & Shahid Shahidul Islam Hall, RUET \\
3 & S3 & Tin Shed Hall, RUET \\
4 & S4 & Selim Hall, RUET \\
5 & S5 & Shahid Ziaur Rahman Hall, RUET \\
6 & S6 & Sheikh Hasina Hall, RUET \\
7 & S7 & Main Tank, RUET \\
8 & S8 & Sere Bangla Hall, RU \\
9 & S9 & Bangabandhu Hall, RU \\
10 & S10 & Habibur Rahman Hall, RU \\
11 & S11 & Mother Bosk's Hall, RU \\
12 & S12 & Sohraurdi Hall, RU \\
13 & S13 & Taposhi Rabeya Hall, RU \\
14 & S14 & Begum Khaleda Zia Hall, RU \\
15 & S15 & Main tank, RU \\
16 & S16 & Palin Ladies Hall, RMC \\
17 & S17 & Pintu Hall, RMC \\
18 & S18 & Main tank, RMC \\
19 & S19 & Muslim Hall, RC \\
20 & S20 & Ladies Hall, RC \\
21 & S21 & Samsuddin Hall, RGDC \\
22 & S22 & Ladies hall, RPI \\
\hline
\end{tabular}

\subsection{Laboratory Experiment}

After collecting, the samples were brought Public Health Engineering Lab, RUET and the concentration of $\mathrm{Fe}$ was determined by calorimetric method using dilute hydrochloric acid, potassium permanganate solution, potassium thiocyanite solution and standard iron solution. The $\mathrm{pH}$, electrical conductivity, BOD, COD and DO were measured by a digital meter (Hach Sension ${ }^{\mathrm{Tm}} 156$ Multiparameter meter).Total alkalinity and hardness was determined by titration with a standard $0.02 \mathrm{~N}$ sulfuric acid solutions and methyl orange as indicator. The concentration of Mn was determined by per sulfate method using standard manganese solution and ammonium per sulfate. Chloride was determined by titrimetric method using $0.05 \mathrm{M} \mathrm{AgNO}_{3}$ solution in presence of potassium chromate $(10 \%)$ indicator. Total Acidity and $\mathrm{CO}_{2}$ was determined by titrimetric method using standard $0.02 \mathrm{~N} \mathrm{NaOH}$ and phenolphthalein as indicator. The concentration of as was determined by using NIPSOM Kit box is a modified kit of ANN. The color of water was determined by using Hellige color Comparator (Levibond 2000). The turbidity of water was measured by TN-100/T-100 Turbidity Meter.

\section{Results and Discussions}

The ground water quality was assessed by analysis of some particular parameters and compared with Bangladesh and WHO standard standards [4,5].

It was observed the $\mathrm{pH}$ varies from 8.2-8.66 and $\mathrm{pH}$ of most samples is within limit. Only some places beyond permissible limit. TS varies from 9.4-565.4 mg/l, TDS varies from 1.01-528 mg/l and TSS varies from 7.2-9.7 mg/l. Most of the samples contain TS and TDS beyond both Bangladesh and WHO standard, but TSS in most samples within these standard limit.

It was observed that color varies from 0-4.8 pt-co unit and electric conductivity varies from $210-900 \mu \mathrm{s} / \mathrm{cm}$. Both color and electric conductivity are within standard limit. The concentration of iron varies from $0-0.7 \mathrm{mg} / \mathrm{l}$ and within Bangladesh standard in the entire sample.

The concentration of dissolved $\mathrm{CO}_{2}$ varies from 23-240 $\mathrm{mg} / \mathrm{l}$ and chloride varies from 150-278 $\mathrm{mg} / \mathrm{l}$. The total alkalinity, arsenic and manganese are nil in all location. The hardness varies from $190-460 \mathrm{mg} / \mathrm{l}$ and turbidity varies from 0.5-9 NTU. The total acidity varies from $1-8 \mathrm{mg} / \mathrm{l}$. DO varies from $0.3-19 \mathrm{mg} / \mathrm{l}, \mathrm{BOD}$ varies from $0.1-1.5 \mathrm{mg} / \mathrm{l}$ and 
COD varies from 1.2-5.7 $\mathrm{mg} / \mathrm{l}$. The fecal coliform varies from 6-11 cfu/100ml and the total coliform varies from 59-106 cfu/100ml. Total coliform and fecal coliform are excessive in water of Palin Ladies Hall and Pintu Hostel in Rajshahi Medical College.

Table 2. PH, TS, TDS, TSS, color and EC of sample water

\begin{tabular}{lllllll}
\hline $\begin{array}{l}\text { Sample } \\
\text { Name }\end{array}$ & $\mathbf{p H}$ & $\begin{array}{l}\text { TS } \\
(\mathbf{m g} / \mathbf{l})\end{array}$ & $\begin{array}{l}\text { TDS } \\
(\mathbf{m g} / \mathbf{l})\end{array}$ & $\begin{array}{l}\text { TSS } \\
(\mathbf{m g} / \mathbf{l})\end{array}$ & $\begin{array}{l}\text { Color } \\
(\mathbf{p t}-\mathbf{c o} \\
\mathbf{u n i t})\end{array}$ & $\begin{array}{l}\text { EC } \\
(\boldsymbol{\mu S} / \mathbf{c m})\end{array}$ \\
\hline S1 & 8.5 & 537.6 & 528 & 9.6 & 4.5 & 399 \\
S2 & 8.62 & 463.6 & 454.4 & 9.2 & 3.8 & 230 \\
S3 & 8.4 & 383.6 & 377.3 & 9.3 & 3.8 & 250 \\
S4 & 8.52 & 403.2 & 393.6 & 9.4 & 4.5 & 280 \\
S5 & 8.32 & 565.4 & 555.8 & 9.6 & 2.8 & 210 \\
S6 & 8.35 & 408 & 398.3 & 9.7 & 4.8 & 495 \\
S7 & 8.52 & 483 & 473.5 & 9.5 & 3.5 & 467 \\
S8 & 8.5 & 409.4 & 401.3 & 8.1 & - & 789 \\
S9 & 8.62 & 419 & 409.7 & 8.62 & - & 876 \\
S10 & 8.45 & 215.4 & 206.4 & 9 & - & 498 \\
S11 & 8.52 & 400 & 390.4 & 9.2 & - & 789 \\
S12 & 8.32 & 360 & 351.2 & 8.8 & - & 790 \\
S13 & 8.35 & 360 & 351.4 & 8.6 & - & 704 \\
S14 & 8.52 & 457.8 & 450.1 & 8.7 & - & 756 \\
S15 & 8.2 & 332.4 & 324.4 & 8 & - & 876 \\
S16 & 8.66 & 259.6 & 252 & 7.6 & - & 789 \\
S17 & 8.49 & 367.6 & 360.1 & 7.5 & - & 698 \\
S18 & 8.49 & 253.6 & 246.4 & 7.2 & - & 790 \\
S19 & 8.24 & 217.2 & 208.9 & 8.6 & - & 900 \\
S20 & 8.24 & 537.6 & 528 & 8.54 & - & 400 \\
S21 & 8.52 & 71.2 & 62.9 & 8.3 & - & 456 \\
S22 & 8.24 & 9.4 & 1.01 & 8.4 & - & 867 \\
Bangladesh & $6.5-8.5$ & 10 & 1000 & 500 & 15 & 1200 \\
Standard & & & & & & \\
WHO & $6.5-8.5$ & - & 1000 & 80 & 15 & 1200 \\
Standard & & & & & & \\
\hline & & & & & &
\end{tabular}

Table 3. Concentration of arsenic, iron, manganese, chloride and dissolved $\mathrm{CO}_{2}$ in sample water

\begin{tabular}{|c|c|c|c|c|c|}
\hline $\begin{array}{l}\text { Sample } \\
\text { Name }\end{array}$ & $\begin{array}{l}\text { As } \\
(\mathrm{mg} / \mathrm{l})\end{array}$ & $\begin{array}{l}\mathrm{Fe} \\
(\mathrm{mg} / \mathrm{l})\end{array}$ & $\begin{array}{l}\text { Mn } \\
(\mathrm{mg} / \mathrm{l})\end{array}$ & $\begin{array}{l}\text { Chloride } \\
\text { (mg/l) }\end{array}$ & $\begin{array}{l}\text { Dissolved } \\
\mathrm{CO}_{2} \\
(\mathrm{mg} / \mathrm{l})\end{array}$ \\
\hline S1 & - & 0.3 & - & 150 & 28 \\
\hline $\mathrm{S} 2$ & - & - & - & 165 & 32 \\
\hline S3 & - & 0.4 & - & 171 & 23 \\
\hline S4 & - & - & - & 189 & 35 \\
\hline S5 & - & 0.3 & - & 185 & 32 \\
\hline S6 & - & 0.6 & - & 180 & 31 \\
\hline S7 & - & 0.3 & - & 170 & 32 \\
\hline S8 & - & 0.1 & - & 210 & 28 \\
\hline S9 & - & 0.3 & - & 220 & 32 \\
\hline S10 & - & - & - & 205 & 23 \\
\hline S11 & - & 0.4 & - & 208 & 35 \\
\hline $\mathrm{S} 12$ & - & 0.7 & - & 230 & 32 \\
\hline S13 & - & 0.2 & & 230 & 28 \\
\hline S14 & - & 0.3 & - & 204 & 40 \\
\hline S15 & - & - & - & 206 & 40 \\
\hline S16 & - & - & - & 160 & 240 \\
\hline S17 & - & - & - & 156 & 220 \\
\hline S18 & - & - & - & 178 & 210 \\
\hline S19 & - & - & - & 212 & 87 \\
\hline S20 & - & - & - & 218 & 88 \\
\hline $\mathrm{S} 21$ & - & - & - & 230 & 18 \\
\hline $\mathrm{S} 22$ & - & - & - & 278 & 18 \\
\hline $\begin{array}{l}\text { Bangladesh } \\
\text { Standard }\end{array}$ & 0.05 & $0.3-1.0$ & 0.1 & $150-600$ & $25-50$ \\
\hline $\begin{array}{l}\text { WHO } \\
\text { Standard }\end{array}$ & 0.01 & 0.3 & 0.1 & 25 & 30 \\
\hline
\end{tabular}

Table 4. Hardness, total alkalinity, total acidity and turbidity of sample wate:

\begin{tabular}{|c|c|c|c|c|}
\hline $\begin{array}{l}\text { Sample } \\
\text { Name }\end{array}$ & $\begin{array}{l}\text { Hardness } \\
(\mathrm{mg} / \mathrm{l})\end{array}$ & $\begin{array}{l}\text { Total } \\
\text { Alkalinity } \\
\text { (mg/l) }\end{array}$ & $\begin{array}{l}\text { Total } \\
\text { Acidity } \\
\text { (mg/l) }\end{array}$ & $\begin{array}{l}\text { Turbidity } \\
\text { (NTU) }\end{array}$ \\
\hline S1 & 270 & - & 3 & 6.5 \\
\hline S2 & 273 & - & 6 & 8.5 \\
\hline S3 & 271 & - & 3 & 9 \\
\hline S4 & 280 & - & 4 & 7.5 \\
\hline S5 & 260 & - & 1 & 6.5 \\
\hline S6 & 268 & - & 5 & 7 \\
\hline S7 & 270 & - & 2 & 9 \\
\hline S8 & 200 & - & 3 & 2 \\
\hline S9 & 202 & - & 2 & 3.5 \\
\hline S10 & 198 & - & 3 & 1.3 \\
\hline S11 & 212 & - & - & 0.7 \\
\hline $\mathrm{S} 12$ & 212 & - & 1 & 0.9 \\
\hline S13 & 208 & - & 2 & 0.5 \\
\hline S14 & 212 & - & 4 & 2.6 \\
\hline S15 & 204 & - & - & 1.5 \\
\hline S16 & 305 & - & 5 & 6.5 \\
\hline S17 & 408 & - & 8 & 8.6 \\
\hline S18 & 460 & - & 3 & 8.8 \\
\hline S19 & 205 & - & 5 & 4 \\
\hline S20 & 240 & - & 8 & 2 \\
\hline $\mathrm{S} 21$ & 190 & - & 3 & 1.5 \\
\hline S22 & 230 & - & 1 & 0.9 \\
\hline $\begin{array}{l}\text { Bangladesh } \\
\text { Standard }\end{array}$ & $200-500$ & & - & 10 \\
\hline $\begin{array}{l}\text { WHO } \\
\text { Standard }\end{array}$ & 200 & & - & 5 \\
\hline
\end{tabular}

Table 5. DO, BOD, COD, fecal coliform and total coliform of sample water.

\begin{tabular}{|c|c|c|c|c|c|}
\hline $\begin{array}{l}\text { Sample } \\
\text { Name }\end{array}$ & $\begin{array}{l}\text { DO } \\
(\mathrm{mg} / \mathrm{l})\end{array}$ & $\begin{array}{l}\text { BOD } \\
(\mathrm{mg} / \mathrm{l})\end{array}$ & $\begin{array}{l}\text { COD } \\
(\mathrm{mg} / \mathrm{l})\end{array}$ & $\begin{array}{l}\text { Fecal } \\
\text { Coliform } \\
(\mathrm{cfu} / \mathbf{1 0 0 m})\end{array}$ & $\begin{array}{l}\text { Total } \\
\text { Coliform } \\
(\mathrm{cfu} / \mathbf{1 0 0 m l})\end{array}$ \\
\hline $\mathrm{S} 1$ & 0.5 & 0.2 & 3.4 & 7 & 70 \\
\hline S2 & 0.7 & 0.1 & 5.7 & 9 & 67 \\
\hline S3 & 0.4 & 0.4 & 2.1 & 15 & 89 \\
\hline S4 & 0.8 & 0.1 & 2.3 & 10 & 65 \\
\hline S5 & 0.3 & 0.3 & 4.8 & 9 & 78 \\
\hline S6 & 0.8 & 0.3 & 2.3 & 11 & 59 \\
\hline S7 & 0.5 & 0.7 & 2.1 & 8 & 87 \\
\hline S8 & 0.5 & 0.3 & 3.5 & 7 & 60 \\
\hline S9 & 1.2 & 0.5 & 2.5 & 9 & 65 \\
\hline S10 & 0.6 & 0.8 & 5.6 & 11 & 67 \\
\hline S11 & 0.8 & 0.5 & 2.9 & 8 & 75 \\
\hline $\mathrm{S} 12$ & 0.2 & 0.2 & 3.1 & 9 & 71 \\
\hline S13 & 0.7 & 0.1 & 4.8 & 6 & 72 \\
\hline S14 & 0.7 & 0.8 & 2.7 & 7 & 67 \\
\hline S15 & 0.1 & 1.5 & 1.2 & 11 & 65 \\
\hline S16 & 0.1 & 0.2 & 2.1 & 10 & 87 \\
\hline S17 & 0.2 & 0.3 & 1.2 & 12 & 106 \\
\hline S18 & 0.6 & 0.1 & 1.6 & 13 & 112 \\
\hline S19 & 0.5 & 0.4 & 1.4 & 8 & 95 \\
\hline S20 & 0.8 & 0.8 & 1.8 & 9 & 69 \\
\hline $\mathrm{S} 21$ & 1.5 & 0.3 & 3.6 & 7 & 80 \\
\hline S22 & 1.9 & 0.6 & 3.8 & 11 & 89 \\
\hline $\begin{array}{l}\text { Bangladesh } \\
\text { Standard }\end{array}$ & 6 & 0.2 & 4 & 0 & 0 \\
\hline $\begin{array}{l}\text { WHO } \\
\text { Standard }\end{array}$ & - & - & - & - & - \\
\hline
\end{tabular}




\section{Conclusion}

It may be concluded from the study that the quality of ground water in these educational institutions is in general good for utilization in drinking, agriculture, industrial and domestic purposes. However a few water samples are found unsuitable especially due to high contents of total coliform and fecal coliform. The present status should not let continue unconcerned for the situation may get critical in near future. Measures should be taken to regulate the water quality determining parameters as well as to minimize total coliform and fecal coliform contents at best in drinking water. Government and Public Health Departments may play a vital role in these educational institutions.

\section{References}

[1] B. K. Sharma, Environmental Chemistry, 1st ed., 1994.
[2] K. E. Anderson, Water Well Handbook, Missouri Water Drillers Association, USA, 1966.

[3] BWPCB (Bangladesh Water Pollution Control Board), Water Quality Standard, 1976.

[4] WHO (World Health Organization), International for Drinking Water, Palais Des Nations, Geneva, 1967.

[5] L. S. Clesceri, A. E. Greenberg and R. R. Trussel, Standard Method for the Examination of Water and Waste Water, 17th ed., Americal Public Health Asso., Washington D.C. 20005, 1989.

[6] Q. M. Chowdhury, "Water and Environment," Forum of Environmental Journalist of Bangladesh, 2001.

[7] B. D. Tripathi and S. R. Govil, "Water Pollution (An Experimental Approach)," CBS Publicaion, New Delhi, 2001. 